\title{
Polymer crystallization-driven, periodic patterning on carbon nanotubes
}

\author{
Lingyu $\mathrm{Li}^{\dagger}$, Christopher Y. $\mathrm{Li}^{\dagger} *$, ChaoYing $\mathrm{Ni}^{\ddagger}$
}

${ }^{\dagger}$ A. J. Drexel Nanotechnology Institute and Department of Materials Science and Engineering, Drexel

University, Philadelphia, PA 19104

${ }^{\dagger}$ W. M. Keck Electron Microscopy Facility, Department of Materials Science and Engineering, University of Delaware, Newark, DE 19716

* To whom correspondence should be addressed: chrisli@drexel.edu

\section{Experimental section}

\section{Materials:}

All CNTs used in this study are listed in Table 1 of the manuscript. CNTs were washed with 2.4 M nitric acid for 30 mins. The resulting CNTs were then centrifuged, collected and dried in a vacuum oven. Nylon $66\left(\mathrm{M}_{\mathrm{n}}=10,000 \mathrm{~g} / \mathrm{mol}\right)$ was supplied by DuPont Company. Linear polyethylene $(\mathrm{MFI}=12 \mathrm{~g} / 10 \mathrm{~min}), \mathrm{p}$-xylene, 1,2-diclorobenzene (DCB), and glycerin were purchased from Aldrich and used as received. 


\section{Experimental procedure:}

Functionalized CNTs were obtained via solution crystallization using CNTs as seeds. Scheme S.1 shows a typical experimental route. For polyethylene solution crystallization, both $p$-xylene and DCB were used as solvent. $0.5 \mathrm{mg}$ PE was dissolved in $4 \mathrm{~g}$ p-xylene at $120{ }^{\circ} \mathrm{C} .0 .1 \mathrm{mg}$ MWNT-10 and $1 \mathrm{~g} p$-xylene solution was sonicated for $2-3 \mathrm{hrs}$ at $45^{\circ} \mathrm{C}$ and then added to $\mathrm{PE} / \mathrm{p}$ -

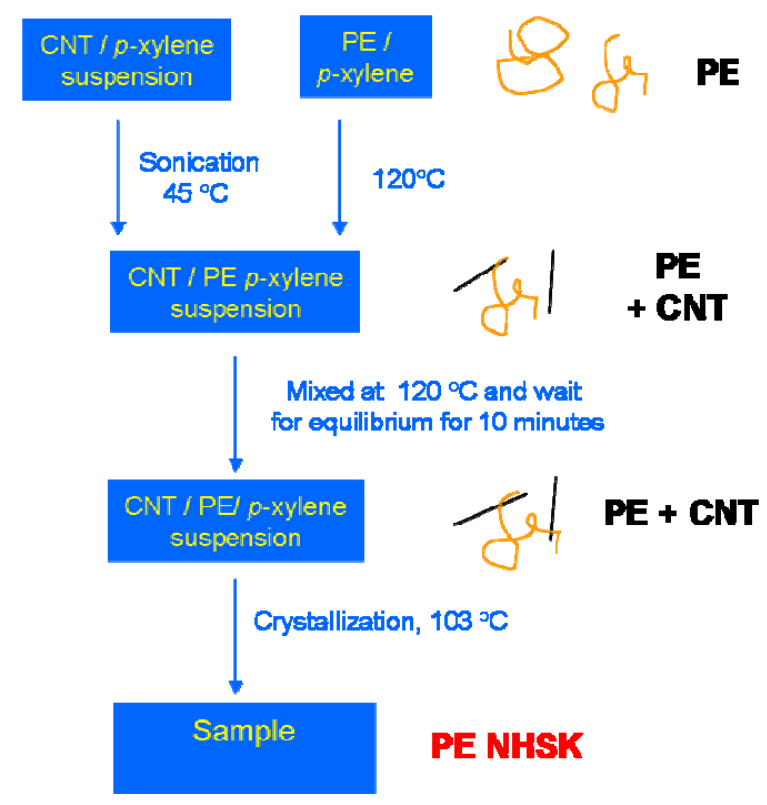

Scheme S.1 Formation process of PE/NHSK.

xylene solution. The mixture was then quenched to the preset crystallization temperature $\left(\mathrm{T}_{\mathrm{c}}=\right.$ $\left.103{ }^{\circ} \mathrm{C}\right)$. The crystallization time was controlled to be $0.5-3 \mathrm{hrs}$. Sample was isothermally filtered after crystallization to remove the uncrystallized materials. When DCB was used as the solvent of PE crystallization, SWNT concentration was varied from $0.01 \mathrm{wt} \%-0.1 \mathrm{wt} \%$. $\mathrm{T}_{\mathrm{c}}$ was varied from $87-91{ }^{\circ} \mathrm{C}$. For Nylon 66 solution crystallization, glycerin was used as solvent and the 
concentration was $\sim(\mathrm{w} / \mathrm{w}) 0.01 \%$. $\mathrm{T}_{\mathrm{c}}$ used for MWNT-10 and SWNT was 185 and $188^{\circ} \mathrm{C}$, respectively. TEM experiments were conducted using a JEOL-2000 FX microscope with an accelerating voltage of $120 \mathrm{kV}$. NHSK suspensions were collected on a carbon-coated TEM grid. Both Pt-shadowed and non-shadowed samples were used. SEM experiments were carried out using a FEI/Phillips XL30 field emission environmental SEM and the acceleration voltage was $15 \mathrm{kV}$. NHSK suspensions were collected on a glass slide. After solvent evaporation, NHSKs were sputtered with Pt for 25 seconds before SEM observation. Raman spectra were acquired with a Raman micro spectrometer (Renishaw 1000) using an Ar ion laser (514.5 nm) in backscattering geometry. A 50x objective with a spot size of $\sim 1 \mu \mathrm{m}$ in diameter was used. A low laser power density was used on the sample (laser power $<2 \mathrm{~mW}$ ) to avoid laser heating of the tubes. Wide angle X-ray diffraction (WAXD) was carried out using a Siemens D-500 X-Ray Diffractometer with $\mathrm{Cu}$ Ka radiation $\lambda=0.15406 \mathrm{~nm}$ at $40 \mathrm{kV}$ and $30 \mathrm{~mA}$. Data were collected over the $2 \theta$ range $15-30^{\circ}$ with a step size of $0.016^{\circ}$. Differential Scanning Calorimetry (DSC) was carried out uisng a Perkin-Elmer DSC7 at a heating and cooling rate of $10{ }^{\circ} \mathrm{C} / \mathrm{min}$ under a nitrogen atmosphere. Sample with a mass of $3.6 \mathrm{mg}$ were encapsulated in sealed aluminum pans. The temperature and heat flow were calibrated using standard materials (Indium and Zinc) at different cooling and heating rates between 5 and $40{ }^{\circ} \mathrm{C} / \mathrm{min}$. 

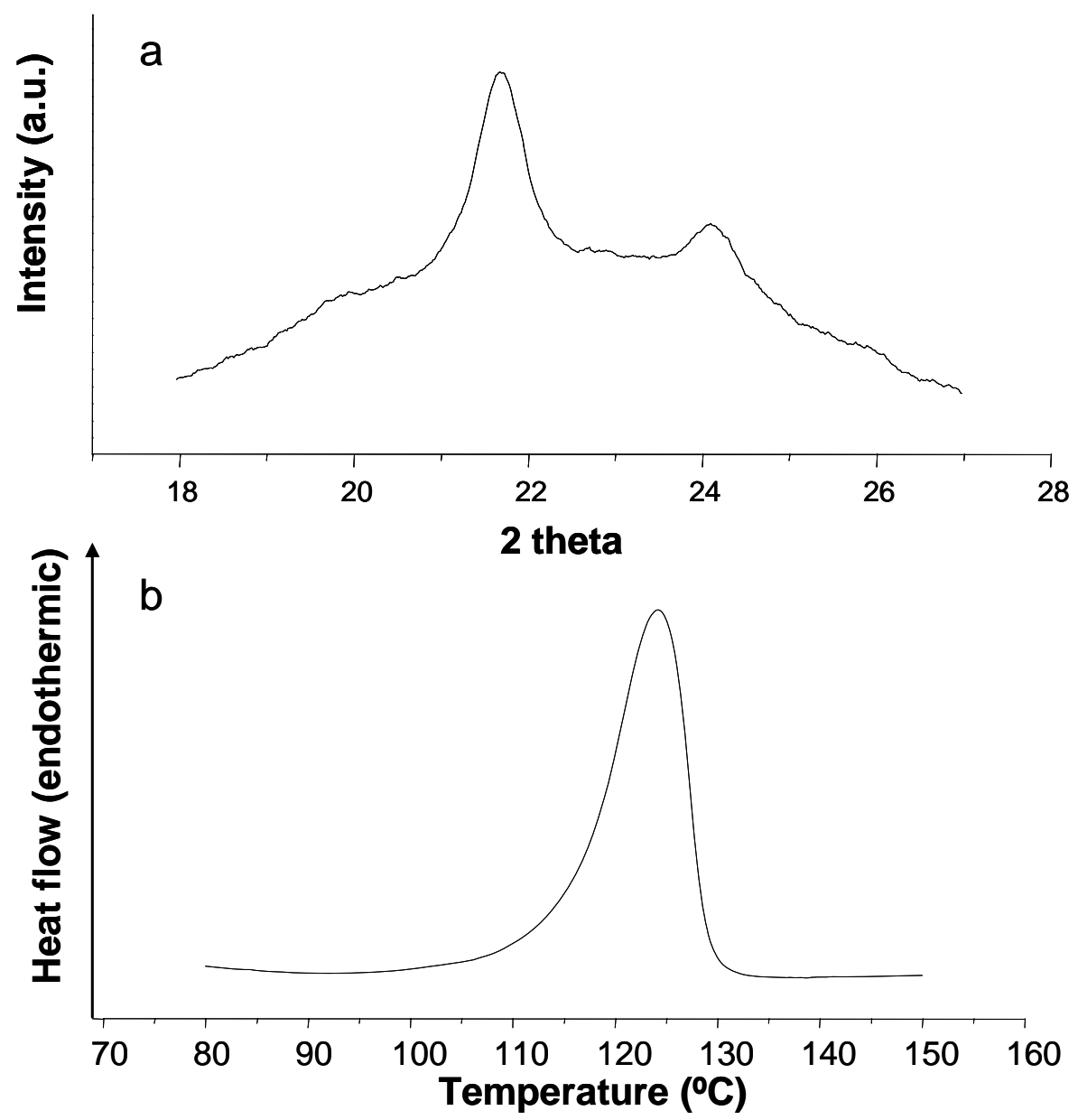

Figure S.1. (a) WAXD pattern and (b) DSC thermogram of PE/SWNT NHSKs. WAXD pattern clearly shows the (110) and (200) diffraction peaks of crystalline PE and DSC data shows the melting endothermic peak of PE single crystals. 


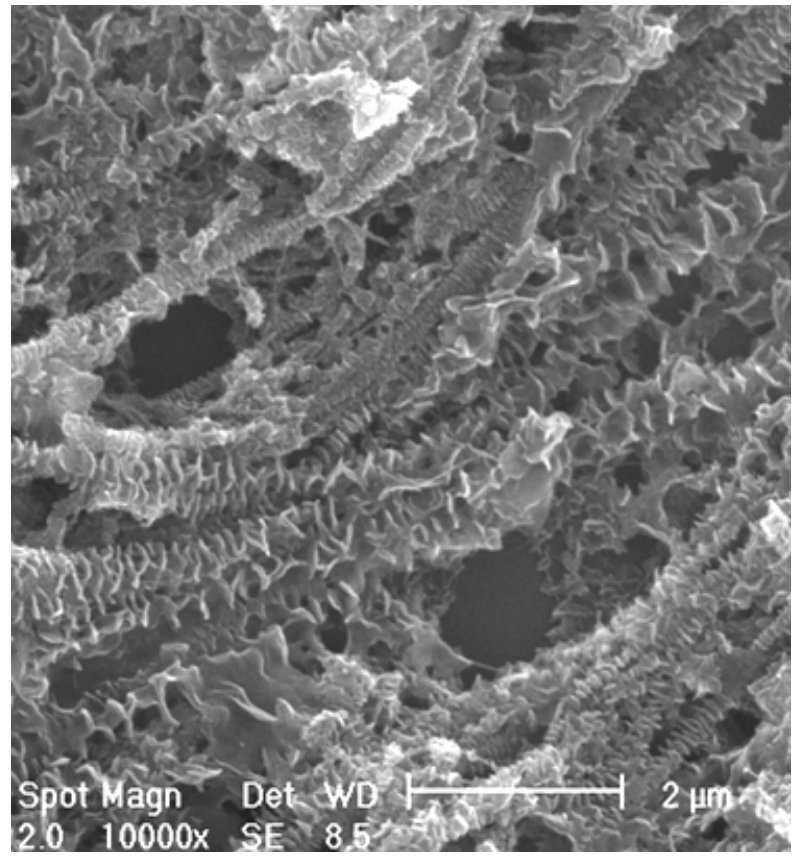

Figure S.2 SEM image of PE/ MWNT-10 NHSK prepared in p-xylene with relatively high PE concentration. The PE and SWNT concentrations are $0.02 \mathrm{wt} \%$ and $0.002 \mathrm{wt} \%$, respectively. Kebabs are much large, which could ensure high degree of CNT functionalization. 


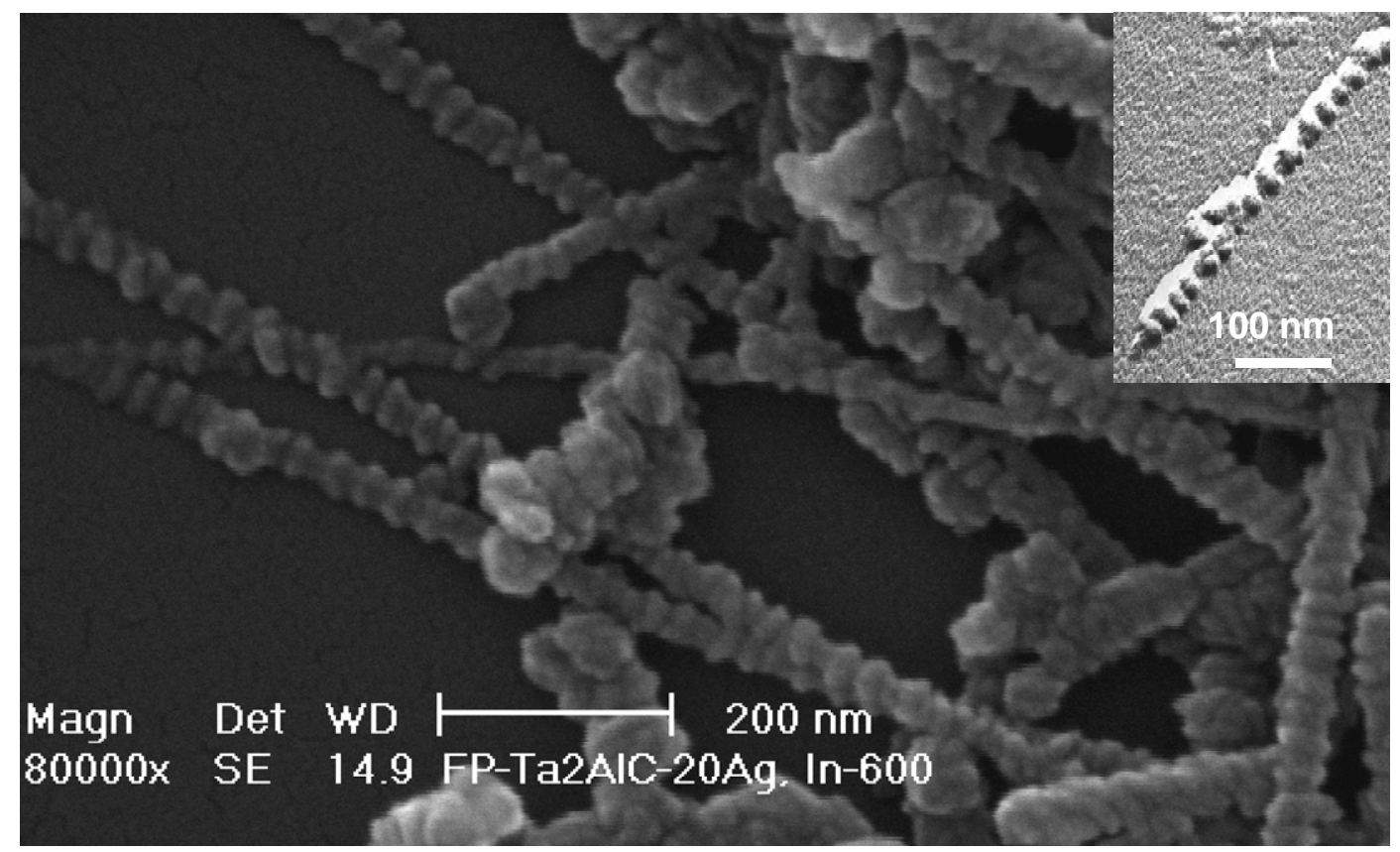

Figure S.3 Nylon 66/MWNT NHSK structures produced by crystallization of Nylon 66 on MWNT-10s at $185{ }^{\circ} \mathrm{C}$ in glycerol for $0.5 \mathrm{hr}$. The PE, SWNT and MWNT concentrations are $0.01 \mathrm{wt} \%, 0.002 \mathrm{wt} \%$ and $0.002 \mathrm{wt} \%$, respectively. The SEM image shows that all the MWNTs are separated and periodically decorated by Nylon 66 single crystals. Inset shows a TEM image of Nylon 66/SWNT NHSK structure. The TEM sample was shadowed with Pt to enhance the contrast. 